MATEC Web of Conferences 3, 01062 (2013)

DOI: $10.1051 /$ matecconf/20130301062

(C) Owned by the authors, published by EDP Sciences, 2013

\title{
Nanosized objects in equilibrium supercritical fluids
}

\author{
B. Sedunov \\ Computer and Information Systems Department, Russian New University, Moscow 105005, Russia
}

\section{Introduction}

Supercritical fluids (SCF) now attract a large attention of both practitioners and researchers. They open new possibilities for dry cleaning, processing of chemical and nuclear wastes, residuum oil supercritical extraction and selective extraction of useful substances from biomasses and mineral ores [1-3]. From a scientific point of view they present a new state of matter, intermediate between gases and liquids.

The growing utilization of SCF in modern chemical technologies is based on the remarkable mixture of solvent power, characteristic to liquids, and transport properties, specific for gases. This mixture of features specific for liquids and gases in SCF is due to their high heterogeneity resulting from the plurality of nanosized clusters in the gas-like zone of the fluid and nanosized pores in the liquid-like zone.

Supercritical fluids near their critical density possess huge density fluctuations. The well known and seen by eyes critical opalescence results from heterogeneities in fluids near the critical point with dimensions measured in microns [4]. But the visible transparency of the supercritical fluid over the critical point does not mean that the heterogeneities have disappeared. Their dimensions simply moved from the micro- to nano- scale [5].

Dr. K. Nishikawa and her group by X-rays scattering have discovered the peak line of the density fluctuations in SCF [6-8]. They named the peak line on the (T, P) diagram as the ridge. This line may be traced also by peaks of some other thermophysical properties, especially, the constant pressure heat capacity $[9,10]$. In many works this peak line is named as the Widom line $[11,12]$. This line divides the supercritical zone into two regions that "can be identified by different dynamical regimes: gas-like and liquid-like" [12]. The ridge or the Widom line looks like the extension of the saturation curve in the supercritical zone.

The aim of this report is the investigation of the nanosized clusters and their characteristics near the ridge line in the gas-like SCF. The investigation is performed by the computer aided analysis of precise equilibrium thermophysical data for several pure van der Waals and polar fluids. The source of data is the NIST database [13], generalizing experimental results of many scientific groups from all over the World. The knowledge of the clusters' characteristics may be useful for practical applications and better understanding of the supercritical fluids' nature.

\section{The method of analysis}

The method is based on the series expansion of the potential energy density by powers of the monomer fraction density $[14,15]$. The molar potential energy $U$ of the fluid is defined here as the pressure dependent part of the molar internal energy $\mathrm{E}$ :

$\mathrm{U}=\mathrm{E}(\mathrm{T}, \mathrm{P})-\mathrm{E}(\mathrm{T}, 0)$

where $\mathrm{E}(\mathrm{T}, \mathrm{P})$ is the internal energy at the existing pressure $\mathrm{P}$ and $\mathrm{E}(\mathrm{T}, 0)$ - at zero pressure. Figure 1 shows the pressure dependence of the $\mathrm{U}(\mathrm{P})$ in supercritical heavy Water, $\mathrm{D}_{2} \mathrm{O}$, at $\mathrm{T}=650 \mathrm{~K}$.

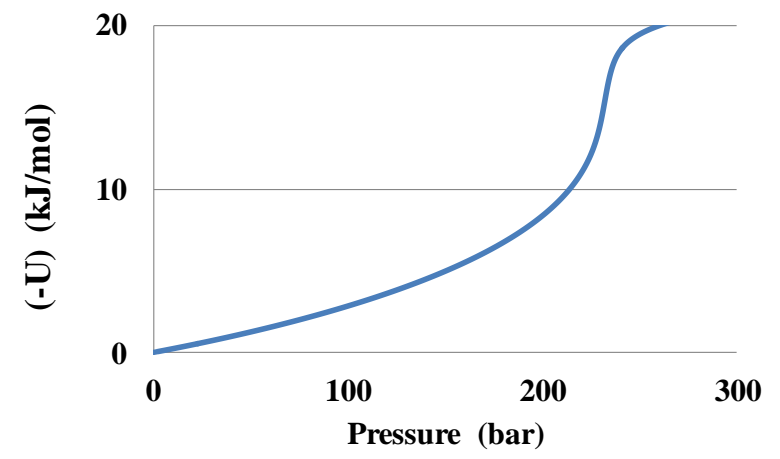

Figure 1. The pressure dependence of the molar potential energy $\mathrm{U}(\mathrm{P})$ in $\mathrm{D}_{2} \mathrm{O}$ at $\mathrm{T}=650 \mathrm{~K}$.

Near zero P the potential energy of gases is zero because monomers, which are the only components of ideal gases, do not interact with each other. The slope of the $U(P)$ curve at small $\mathrm{P}$ is determined mainly by the dimer fraction, but at larger pressures the steepness of slope grows, thus reflecting the contribution of larger clusters. At the ridge $\mathrm{P}_{\mathrm{r}}(\mathrm{T})$ there is a bend of the curve and over it the steepness of slope diminishes due to the domination of repulsions over attractions between molecules.

Instead of the molar potential energy $U$ we expand by powers of the monomer fraction density $\mathrm{D}_{\mathrm{m}}$ the potential 
energy density UD (the potential energy of a unit volume). In pure real gases it is a sum of cluster fractions' contributions (UD) $)_{\mathrm{n}}$ :

$\mathrm{UD}=\sum(\mathrm{UD})_{\mathrm{n}}=-\sum \mathrm{E}_{\mathrm{n}} \mathrm{D}_{\mathrm{n}}$

Here $D_{n}$ are the molar densities and $E_{n}$ - the effective bond energies of n-particle cluster fractions. The series expansion of UD starts from the second power term, because the monomers do not contribute to the potential energy. It is useful for further series expansion to divide UD by $\mathrm{D}_{\mathrm{m}}{ }^{2}$. The Figure 2 shows a tipical view of the pair interaction function $\mathrm{W}=-\mathrm{UD} / \mathrm{D}_{\mathrm{m}}{ }^{2}$ for heavy Water at supercritical $\mathrm{T}=650 \mathrm{~K}$.

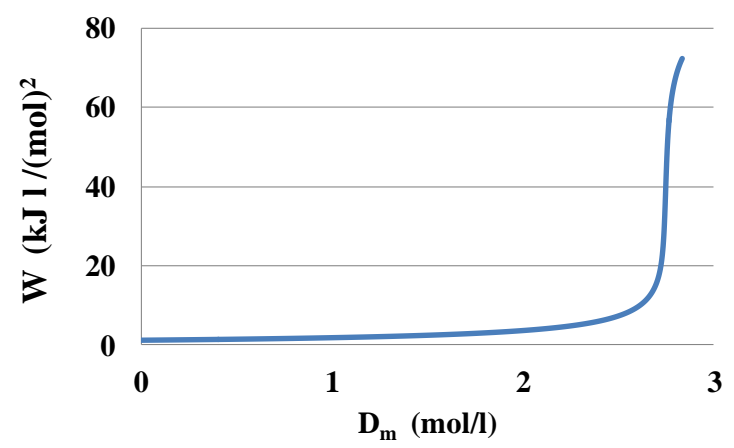

Figure 2. The pair interaction function $\mathrm{W}=U \mathrm{UD} / \mathrm{D}_{\mathrm{m}}{ }^{2}$ versus $\mathrm{D}_{\mathrm{m}}$ in $\mathrm{D}_{2} \mathrm{O}$ at $\mathrm{T}=650 \mathrm{~K}$.

At $\mathrm{D}_{\mathrm{m}}<1 \mathrm{~mol} / \mathrm{l}$ the $\mathrm{W}$ values perform only a slight change from the initial value $\mathrm{W}_{0}$, equal to the second expansion coefficient of UD by $\mathrm{D}_{\mathrm{m}}$, characterizing the dimers. But at $\mathrm{D}_{\mathrm{m}}>2 \mathrm{~mol} / \mathrm{l}$ the $\mathrm{W}$ values accelerate their growth, thus reflecting the growth both of the partial densities and the pair bond numbers for larger cluster fractions. This quick growth increases errors for estimated characteristics of larger clusters and puts severe limitations on the digital processing algorithms.

The clusters' bond energies $E_{n}$ are the averaged energies of their disintegration to monomers. We find the series expansion coefficients $K_{u n}(T)=-(U D)_{n} / D_{m}{ }^{n}$ and plot the logarithm of the $K_{u n}(T)$ versus the reverse temperature. For the zone of $\mathrm{T}$ with a linear graph, its slope presents the cluster bond energy in K (Figure 3).

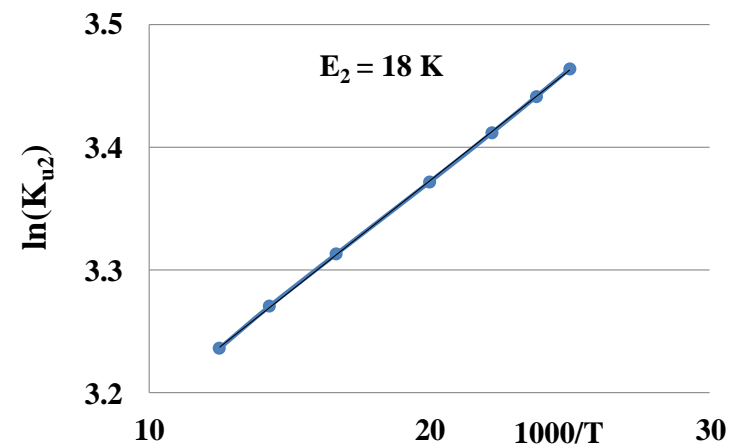

Figure 3. Estimation of the pair bond energy for gaseous $\mathrm{Ne}\left(\mathrm{E}_{2}\right.$ $=18 \mathrm{~K}$ ) at temperatures $40-80 \mathrm{~K}$.

Having found the cluster's bond energy $\mathrm{E}_{\mathrm{n}}$, we can find the equilibrium constant:
$\mathrm{C}_{\mathrm{un}}=\mathrm{K}_{\mathrm{un}} / \mathrm{R} \mathrm{E}_{\mathrm{n}}$

The index " $u$ " means that this equilibrium constant had been found from the potential energy, but not from the other thermophysical property or not from spectroscopy.

\section{The supercritical fluids'structure}

The supercritical fluid is a mixture of zones with highly different local densities of pair bonds. In the gas-like structure there are clusters dissolved in the monomer fraction, but in the liquid-like structure there are pores surrounded by a liquid continuum. The distribution of intermolecular distances in supercritical and near critical fluids is not at all uniform. The models that intuitively consider the low density fluid as the medium with uniformly enlarged intermolecular distances are wrong! We should consider the low density foam-like fluid or the high density fog-like gas as the media with two kinds of molecular complexes: short-distance and long-distance, Figure 4.

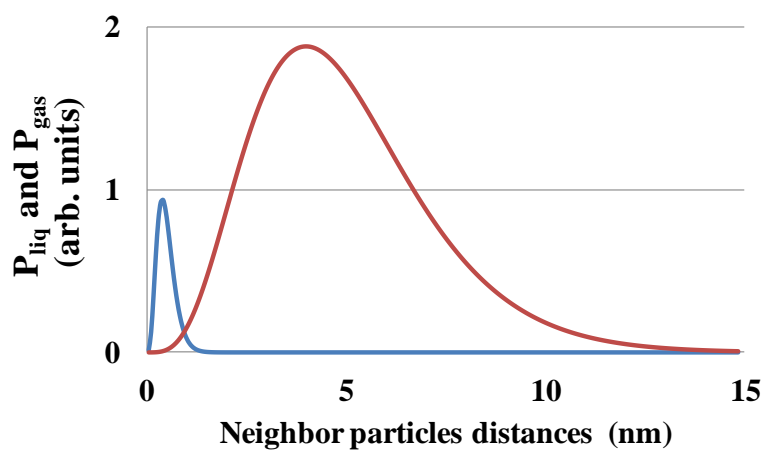

a)

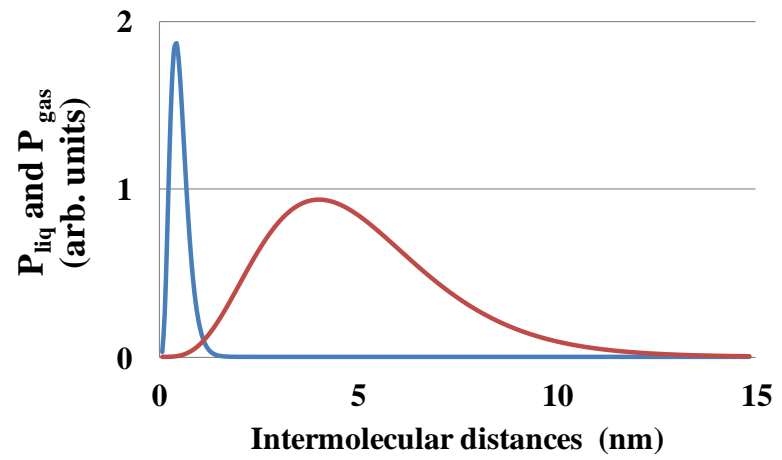

b)

Figure 4. Schematic distribution of probabilities for intermolecular distances in SCF:

a) - a fog-like gas; b) - a foam-like fluid.

Clusters and liquid continuum - blue lines; Monomers and gas in pores - red lines.

The difference between the fog-like gas and the foam-like fluid is not only in the probability values of long and short intermolecular distances, but in the topology of these media. The topology of the fog-like gas is characterized by the monomer fraction continuum with inclusions of dimers and larger clusters, but the topology of the foam-like fluid is the liquid continuum surrounding the gaseous pores $[9,10]$. 
The transition from the fog-like gas to the foam-like fluid in the supercritical zone is not accompanied neither by the change of the total density, nor by the change of the total potential energy. Therefore, this transition differs from the first order phase transition. The author previously was wrong considering it as a phase transition [16], but later changed the name of this transition to the "Soft Structural Transition" [17].

But it is not also the second order transition, because the derivatives of thermophysical values do not have abrupt changes. So, this transition is the transition of a new type: the transition between two topologically different structures [9, 10]. At the ridge pressure the average densities of both structures become equal and there coexist large regions with the fog-like structure and large regions with the foam-like structure. It gives rise to the giant fluctuations of density, directly measured in works of Dr. K. Nishikawa and her group [6-8].

The absence of abrupt changes in the total density and total potential energy makes the boundary between the fog-like and foam-like regions in the ridge zone indistinct. Different experimental methods result in slightly differing $\mathrm{P}_{\mathrm{r}}(\mathrm{T})$ curves forming together the zone of the Soft Structural Transition in the supercritical region that is remarkable due to its outstanding properties. This zone is interesting both for scientific research of phase equilibrium and for practical needs, as the zone of large and quick changes of thermophysical properties. This zone is characterized by large heterogeneity of density and inherits the properties of the near critical region.

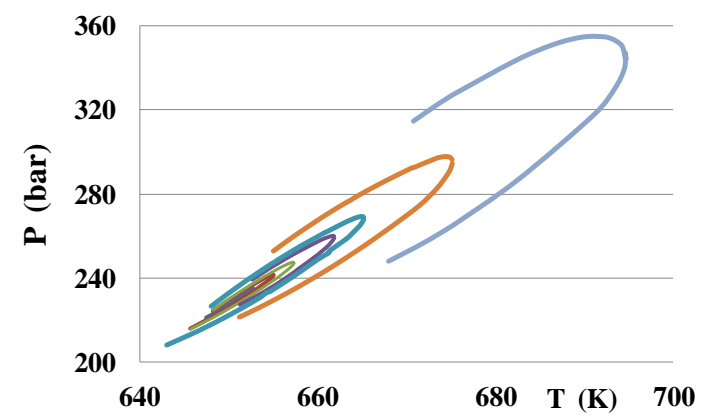

a)

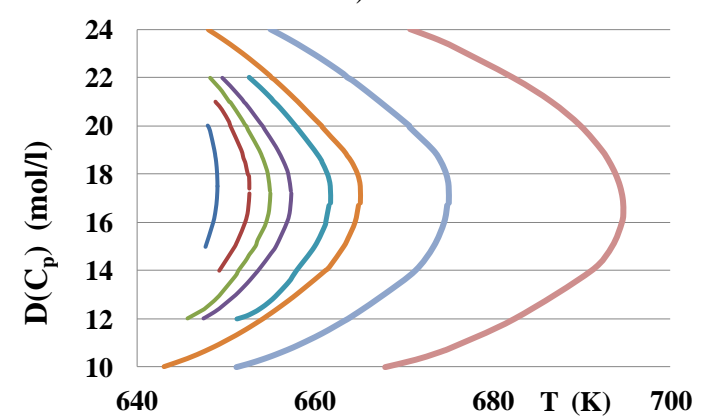

b)

Figure 5. The ridge in Water in coordinates T-P, a), and T-D, b), built by lines of constant $C_{p}$ equal to: $10000,3000,2000$, $1500,1000,800,500,300 \mathrm{~J} / \mathrm{molK}$.

The experimentally found ridge line $\mathrm{P}_{\mathrm{r}}(\mathrm{T})$ depends on the thermophysical property used to find this line. The thermophysical properties, like $\mathrm{C}_{\mathrm{p}}(\mathrm{T}, \mathrm{P})$, change smoothly in the ridge zone, Figure 5.

It is interesting to note that at temperatures more than $5 \mathrm{~K}$ over the Water critical temperature, $\mathrm{T}_{\mathrm{cr}}=647.096 \mathrm{~K}$, the maximal $\mathrm{C}_{\mathrm{p}}$ values [13] along the ridge line correspond to a hyperbolic law, Figure 6:

$\mathrm{C}_{\mathrm{p} \text { ridge }}=\mathrm{k} /\left(\mathrm{T}-\mathrm{T}_{\mathrm{cr} *}\right)$

where $\mathrm{k}=13442$ and $\mathrm{T}_{\text {cr* }}=648.306 \mathrm{~K}$ that is slightly over the real critical temperature.

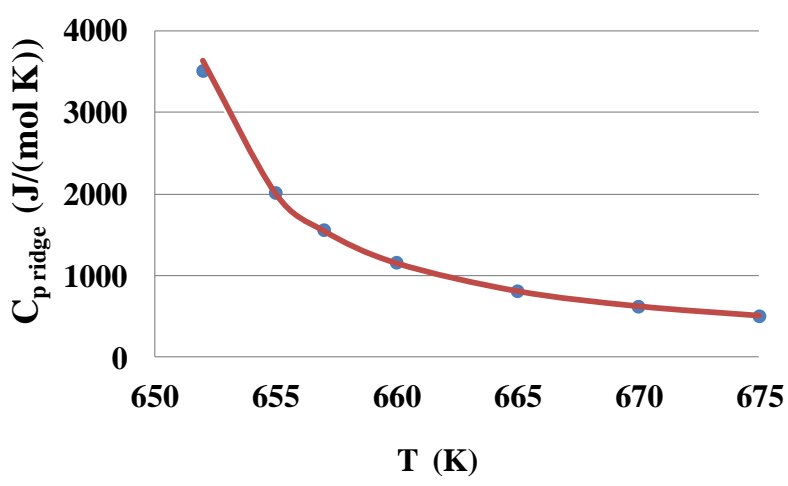

Figure 6. The maximal $\mathrm{C}_{\mathrm{p}}$ values along the ridge line: experimental (blue dots) and model (red line).

It looks like the Curie-Weiss law for magnetic phenomena [18]. The similarity of these so different phenomena cannot be occasional. There should be some physical mechanism responsible for this similarity.

In the ridge region the Speed of Sound has a deep minimum [13], Figure 7. The fall of the Sound Speed in the gaseous phase with the growth of density may be explained by the growth of the average mass of the free moving objects: monomers and clusters.

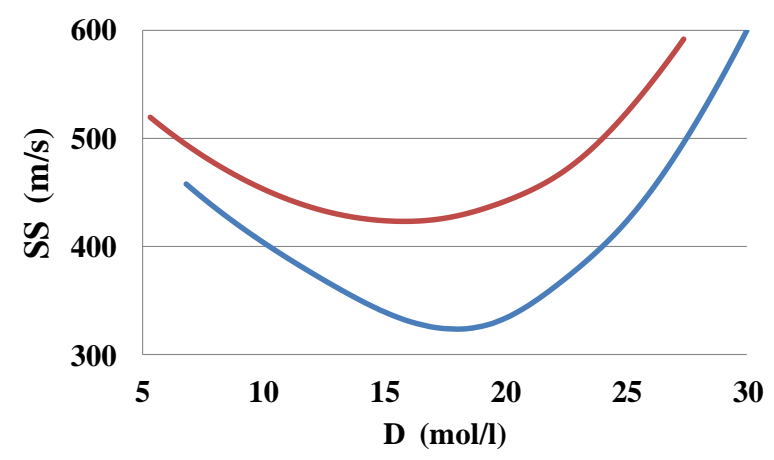

Figure 7. The density dependence of the Speed of Sound in the Water ridge region at temperatures: $652 \mathrm{~K}$ (blue line) and $680 \mathrm{~K}$ (red line).

The growth of the Speed of Sound at the fluid density over the ridge density $D_{r}(T)$ may be explained by the growing role of the intermolecular repulsions in the liquid continuum of the foam-like fluid. 


\section{The clusters'properties}

\subsection{Loose and dense cluster fractions in the fog- like supercritical gases}

Near the ridge the growth of the pair interaction function $\mathrm{W}\left(\mathrm{D}_{\mathrm{m}}\right)$ is so steep, Figure 2, that it looks impossible to come to certain results of the series expansion. But we can subject to series expansion the reverse function $\mathrm{W}_{0} / \mathrm{W}$ instead of the direct $\mathrm{W}$-function [10]. For many investigated gases at supercritical temperatures the $\mathrm{W}_{0} / \mathrm{W}$ $\left(D_{m}\right)$ - function has a long almost linear part of its graph, Figure 8.

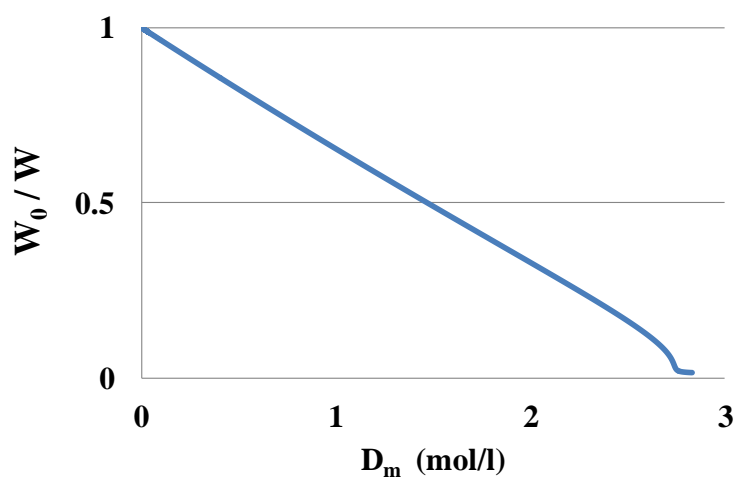

Figure 8. The reverse pair interaction function $\mathrm{W}_{0} / \mathrm{W}$ versus $\mathrm{D}_{\mathrm{m}}$ in $\mathrm{D}_{2} 0$ at $\mathrm{T}=650 \mathrm{~K}$.

This linearity makes easier the expansion process that at small $\mathrm{D}_{\mathrm{m}}$ gives:

$\mathrm{W}_{0} / \mathrm{W}=1-\mathrm{V}_{3} \mathrm{D}_{\mathrm{m}}$

with $V_{3}$ - the temperature dependent effective volume of the new particle attachment to the existing cluster.

In reality, the found values for $\mathrm{V}_{3}$ show a slight linear dependence on $D_{m}$, thus signaling about the dimers' interaction with the existing clusters. But this question should be investigated additionally.

After the first expansion coefficients are found we should return back utilizing the geometric progression equation for the direct function:

$\mathrm{W} / \mathrm{W} 0=1+\mathrm{V} 3 \mathrm{Dm}+(\mathrm{V} 3 \mathrm{Dm}) 2+\ldots$

This expression is valid only for the linear part of the $\mathrm{W}_{0} / \mathrm{W}\left(\mathrm{D}_{\mathrm{m}}\right)$ - function. But we can consider a class of clusters that could correspond to this equation in the expanded zone of its validity. This class of clusters may contain an infinite row of clusters with a universal law of aggregation, given by the $\mathrm{V}_{3}\left(T, \mathrm{D}_{\mathrm{m}}\right)$ function. They seem to be linear chain-like clusters connecting with new particles only at their ends. Their law of aggregation does not depend on the length of the cluster. The $n$-particle clusters of this class have only $(\mathrm{n}-1)$ pair bond, they are loosely bound and can be named as the loose clusters [10]. So, we come to the expression for the loose pair interaction function:

$\mathrm{Wl}=\mathrm{W} 0 /(1-\mathrm{V} 3 \mathrm{Dm})$

If we subtract from the real $\mathrm{W}$ - function the loose $\mathrm{W}_{1}-$ function, we find $\mathrm{W}_{\mathrm{d}}$ - the contribution of the dense 3D clusters in the pair interaction function.

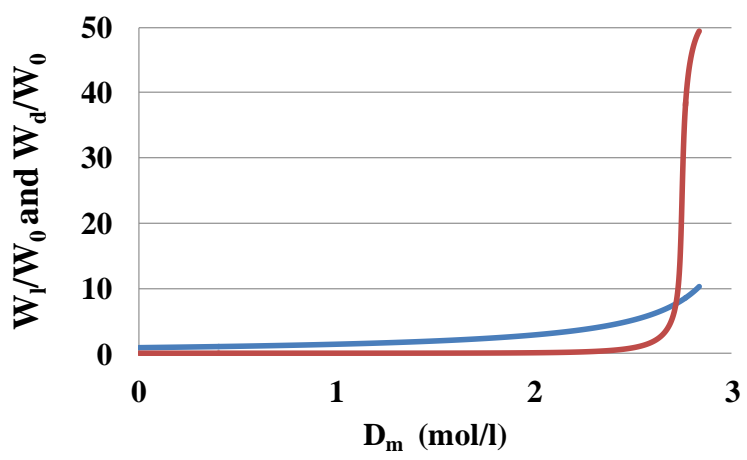

Figure 9. The loose and dense clusters' pair interaction functions: $\mathrm{W}_{\mathrm{l}} / \mathrm{W}_{0}$ (blue line) and $\mathrm{W}_{\mathrm{d}} / \mathrm{W}_{0}$ (red line) in $\mathrm{D}_{2} \mathrm{O}$ at supercritical $\mathrm{T}=650 \mathrm{~K}$.

It is seen that in $\mathrm{D}_{2} \mathrm{O}$ at $\mathrm{T}=650 \mathrm{~K}$ the loose clusters prevail almost in all monomer fraction density range from zero and to slightly under the ridge value. Only in a narrow range near the ridge the contribution of dense clusters essentially overcomes the contribution of loose clusters.

The very steep growth of the pair interaction function for dense clusters seems to prevent from certain results for the series expansion. But the long range of zero values for $\mathrm{W}_{\mathrm{d}}$ opens possibility to divide the $\mathrm{W}_{\mathrm{d}}$ values by the $\mathrm{D}_{\mathrm{m}}{ }^{\mathrm{n} 1}$ and to find the corresponding expansion coefficient $\mathrm{K}_{\mathrm{un} 1}$, where $\mathrm{n}_{1}$ is the starting number of the series expansion for $\mathrm{W}_{\mathrm{d}}$. The numbers of particles in the dense clusters are the numbers of corresponding expansion terms plus 2.

Moving further we find step-by-step the next number $\mathrm{n}_{2}$ and the corresponding contribution in the $\mathrm{W}_{\mathrm{d}}$. But with the growth of the numbers of particles in the dense clusters the processing errors also grow, thus limiting the maximal density with a certain row of the dense clusters' numbers.

For investigated polar and van der Waals gases with a long linear part of the $\mathrm{W}_{0} / \mathrm{W}$ dependence on $\mathrm{D}_{\mathrm{m}}$ the row of dense clusters starts from a relatively large number of particles in the dense cluster and this row is not continuous, but jumping. For example, in Xenon the starting number for dense clusters is 7 and the next numbers at $\mathrm{T}=300 \mathrm{~K}$ are $10,14,26,60,133,150$.

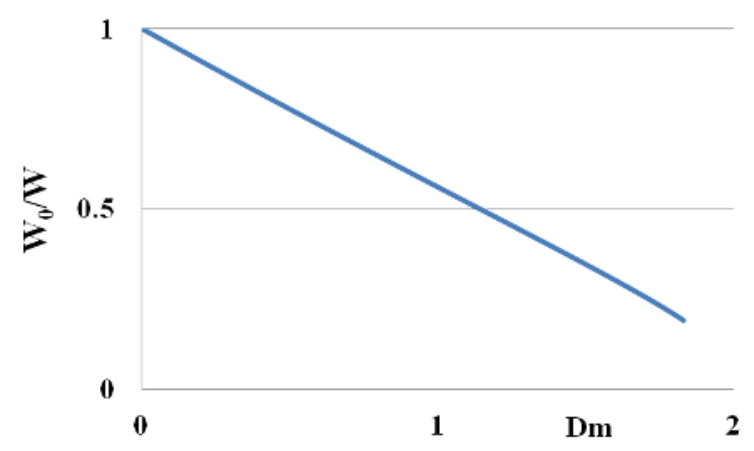

Figure 10. Almost linear reverse pair interaction function $\mathrm{W}_{0} / \mathrm{W}$ for Water at subcritical $\mathrm{T}=600 \mathrm{~K}$. 
In the Water vapor at temperatures near and over the critical temperature the linearity of the $\mathrm{W}_{0} / \mathrm{W}$ dependence on $\mathrm{D}_{\mathrm{m}}$ also takes place, Figure 10.

But at low $\mathrm{T}$ this method does not work because the row of dense clusters starts from a small number 4 for the tightly bonded tetramers in the Water vapor distorting the linearity of the curve, Figure 11.

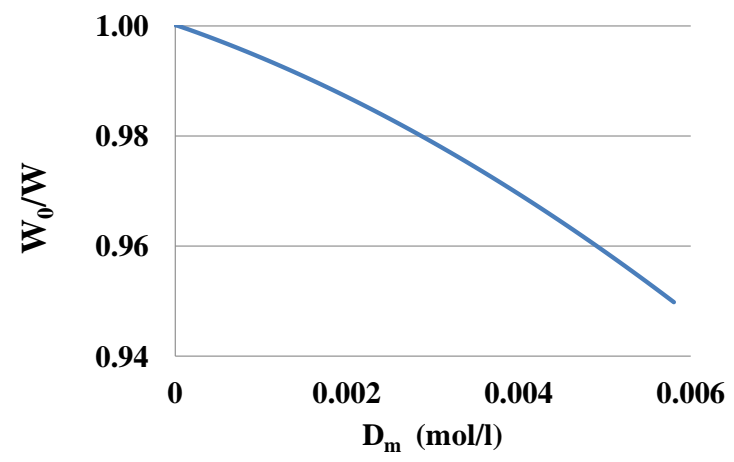

Figure 11. Nonlinear reverse pair interaction function $\mathrm{W}_{0} / \mathrm{W}$ for Water at low $\mathrm{T}=330 \mathrm{~K}$.

\subsection{The results for no polar gases}

For no polar gases the long linear part of the $\mathrm{W}_{0} / \mathrm{W}\left(\mathrm{D}_{\mathrm{m}}\right)$ was demonstrated in [10] for Carbon Dioxide. Here we illustrate this fact on the example of the Xenon high density gas at the supercritical temperature $\mathrm{T}=300 \mathrm{~K}\left(\mathrm{~T}_{\text {cr }}\right.$ $=289.733 \mathrm{~K}$ [13]), Figure 12. Xenon, like the Water vapor, possesses at low $\mathrm{T}$ dense tetramers, but at supercritical temperatures their contribution to the potential energy falls to negligible values and the $\mathrm{W}_{0} / \mathrm{W}\left(\mathrm{D}_{\mathrm{m}}\right)$ dependence becomes linear at small $\mathrm{D}_{\mathrm{m}}$, Figure 12.

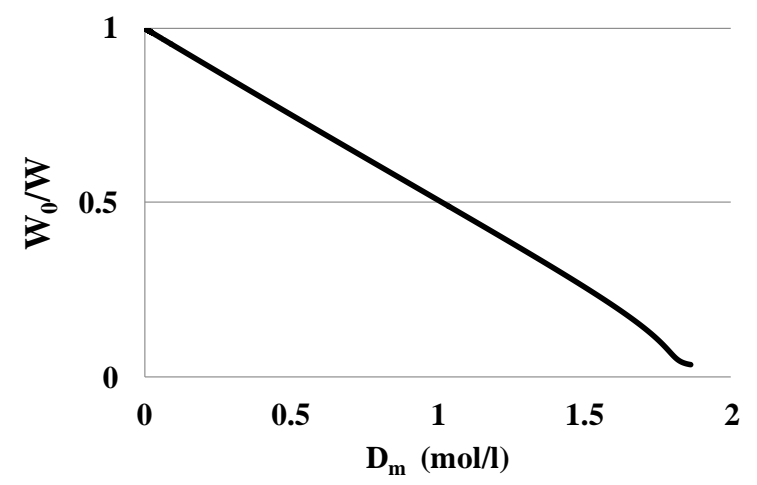

Figure 12. Almost linear reverse pair interaction function $\mathrm{W}_{0} / \mathrm{W}$ for Xenon at $\mathrm{T}=300 \mathrm{~K}$.

The energy of a new particle bonding to the end of the loose cluster $\mathrm{E}_{\text {end }}$ can be found from the temperature dependence of the $\mathrm{W}_{0} / \mathrm{W}$ tangent of slope. This energy is slightly lower than the pair bond energy $E_{2}$, Figure 13.

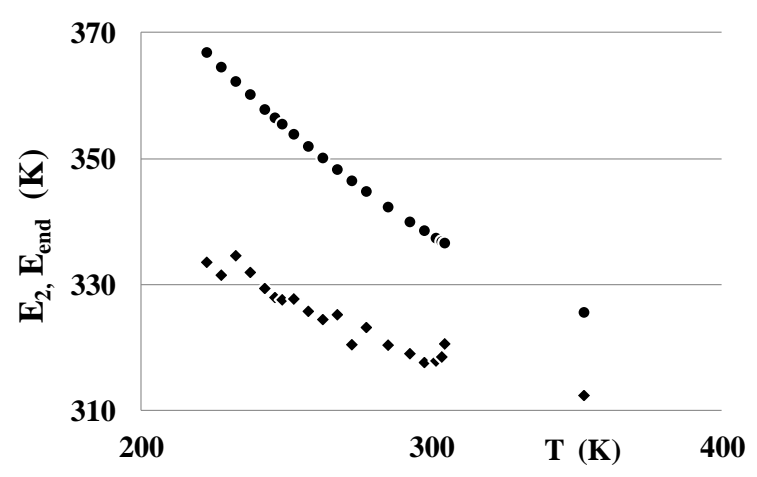

Figure 13. The pair bond energy $\mathrm{E}_{2}$ in the $\mathrm{CO}_{2}$ dimers (round markers) and the energy of a new particle bonding to the end of the linear chain cluster $\mathrm{E}_{\mathrm{end}}$ (rhomb markers).

\section{Conclusion}

- The computer aided processing of precise thermophysical data from the online databases, like the NIST database [13], discovers the cluster structure of real gases at near critical and supercritical temperatures;

- The series expansion of the potential energy density by powers of the monomer fraction density permits to find the bond parameters and equilibrium constants of the cluster fractions;

- At high densities of gases it is possible to find characteristics of linear polymer-like loose clusters with a continuous row of the particle numbers and with a universal bonding mechanism for the end particles;

- The characterization of the loose clusters permits to estimate the properties of the tightly bonded dense clusters with a jumping row of the particle numbers.

- The knowledge of the clusters' characteristics in SCF may be used for optimization of technological processes with SCF.

- Educational perspectives: The idea of the soft structural transition in SCF is very educative. It joins together the idea of continuous gas to liquid conversion and the idea of essential transformation of their structures at this transition.

\section{References}

1. M.A. McHugh, V.J. Krukonis, Supercritical fluid extraction: principles and practice, Butterworth Publishers, Stoneham, MA, USA (1986)

2. L.T. Taylor, Supercritical fluid extraction, John Wiley \& Sons Ltd, New York (1996)

3. Q. Lang, C.M. Wai, Talanta 53771 (2001)

4. M.A. Anisimov, V.A. Rabinovich, V.V. Sychev, Thermodynamics of the Critical State of Individual Substances, CRC Press, Boca Raton (1995)

5. M.A. Anisimov, Thermodynamics at the Meso- and Nanoscale, Encyclopedia of Nanoscience and Nanotechnology, Marcel Dekker, New York (2004)

6. K. Nishikawa and T. Morita, Chemical Physics Letters 316238 (2000)

7. K. Nishikawa et al., Chemical Physics Letters 320 323 (2000) 
8. K. Nishikawa et al., Chemical Physics Letters 368 209 (2003)

9. B. Sedunov, J. of Thermodynamics 2011, Article ID 194353 (2011)

10. B. Sedunov, American J. of Analytical Chemistry 3 899 (2012)

11. G.G. Simeoni et.al. Nature Physics 6503 (2010)

12. S. Han and C.C. Yu, Phys. Rev. E85 051201 (2012)

13. NIST, Thermophysical Properties of Fluid Systems, 2013, http://webbook.nist.gov/chemistry/fluid

14. B. Sedunov, J. of Thermodynamics 2012, Article ID 859047 (2012)

15. B. Sedunov, Int. J. of Thermodynamics 111 (2008)

16. B. Sedunov, 21st IUPAC International Conference on Chemical Thermodynamics, ICCT-2010, Tsukuba, Japan, 2010

17. B. Sedunov, The 22nd International Conference on Chemical Thermodynamics (ICCT) and The 67th Calorimetry Conference (CALCON), Buzios, Brazil, 2012

18. K.H.J. Buschow (Ed.) Concise Encyclopedia of Magnetic and Superconducting Materials, 2nd ed., Elsevier Science, 2005 